\section{Tendência e fatores associados à insegurança alimentar no Brasil: Pesquisa Nacional por Amostra de Domicílios 2004, 2009 e 2013}

\author{
Trends and factors associated with food insecurity \\ in Brazil: the National Household Sample Survey, \\ 2004, 2009, and 2013
}

\section{Tendencia y factores asociados a la inseguridad alimentaria en Brasil: Encuesta Nacional por Muestra de Domicilios 2004, 2009 y 2013}

Taíse Gama dos Santos 1

Jonas Augusto Cardoso da Silveira 1

Giovana Longo-Silva 1

Elyssia Karine Nunes Mendonça Ramires 1 Risia Cristina Egito de Menezes 1

doi: 10.1590/0102-311X00066917

\section{Correspondência}

T. G. Santos

Universidade Federal de Alagoas.

Av. Lourival Melo Mota s/n, Maceió, $A L$

57072-900, Brasil.

taise-gama@hotmail.com

1 Universidade Federal de Alagoas, Maceió, Brasil. segurança alimentar foi avaliada por meio da Escala Brasileira de Insegurança Alimentar. As variáveis independentes foram selecionadas a partir de modelo conceitual de determinação da insegurança alimentar, sendo esse também utilizado para a elaboração dos modelos lineares generalizados múltiplos. Os resultados descrevem tendência de redução na prevalência de insegurança alimentar entre 2004-2013, especialmente, quanto à insegurança alimentar moderada e grave que passou de 17\% (IC95\%: 15,7-18,4) em 2004 para 7,9\% (IC95\%: 7,2-8,7) em 2013. Por outro lado, apesar das importantes reduções na prevalência de insegurança alimentar moderada e grave, observou-se que, independentemente do nível de determinação, os estratos populacionais com menor prevalência em 2004 apresentaram redução relativa de maior magnitude. Quanto aos fatores associados à insegurança alimentar moderada e grave, permaneceram os mesmos nos dez anos cobertos pela PNAD, sendo eles: as macrorregiões Norte/Nordeste, área urbana (na presença de saneamento inadequado), densidade domiciliar $>2$ pessoas/dormitório, possuir $\leq 4$ bens de consumo e a pessoa de referência do domicílio ser do sexo feminino, ter idade < 60 anos, a raça/etnia ser diferente de branca, ter escolaridade $\leq 4$ anos e estar desempregada. Entre 2004-2013, a prevalência de domicílios brasileiros em situação de insegurança alimentar moderada e grave caiu pela metade; contudo, dentro da perspectiva da equidade, destaca-se que os avanços ocorreram de modo desigual, sendo menores nos estratos de maior vulnerabilidade social, econômica e demográfica.

Segurança Alimentar e Nutricional; Fome; Inquéritos Epidemiológicos; Estudos de Séries Temporais 


\section{Introdução}

De acordo com o conceito adotado no país, a segurança alimentar e nutricional representa a "realização do direito de todos ao acesso regular e permanente a alimentos de qualidade, em quantidade suficiente, sem comprometer o acesso a outras necessidades essenciais, tendo, como base, práticas alimentares promotoras de saúde, que respeitem a diversidade cultural e que sejam ambiental, cultural, econômica e socialmente sustentáveis" 1 . A violação desse direito humano conduz à insegurança alimentar, que, no contexto familiar, manifesta-se de forma progressiva, desde a apreensão quanto à disponibilidade futura de comida até a ocorrência da fome 2 . Assim, pelo fato de a insegurança alimentar representar tanto uma ameaça para as condições de saúde e nutrição de uma população quanto problemas estruturais da sociedade, a promoção da segurança alimentar e nutricional foi colocada como um dos objetivos globais de desenvolvimento sustentável 3 .

Mundialmente, estima-se que, aproximadamente, uma em cada nove pessoas ainda vive em situação de insegurança alimentar 4 . Em população residente na Nigéria, a prevalência de insegurança alimentar foi de $61,8 \%$ em 2012 , enquanto que, nos Estados Unidos, uma clássica potência econômica, entre 2005 e 2010, 53,4\% dos adultos de baixa renda relataram insegurança alimentar familiar 6 . Similarmente, no Brasil, diferentes segmentos da população ainda apresentam prevalência elevada de insegurança alimentar, como identificado em domicílios com crianças menores de cinco anos $(45,4 \%) 7$ ou em domicílios localizados em Alagoas (63,7\%), estado com menor Índice de Desenvolvimento Humano (IDH) do país 8 .

Embora seja uma nação emergente, diferentes indicadores confirmam que o Brasil ainda apresenta marcantes desigualdades sociais, pois, embora possua o 75 o maior IDH do mundo $(0,755)$, quando ajustado pela desigualdade, há redução em $26,3 \%$ nesse indicador $(0,557)$. Segundo o índice de Gini, o país ocupa a 12 a posição em termos de desigualdade na distribuição de renda do mundo $(52,7)$, superando o coeficiente de desigualdade humana da América Latina 9.

A exposição à insegurança alimentar também está associada a eventos adversos, como a ocorrência de doenças crônicas, sintomas depressivos e absenteísmo escolar 6,10,11. Por isso, além de violação de direitos humanos, a insegurança alimentar representa importante problema estrutural da sociedade, necessitando ser constantemente monitorado no que se refere aos determinantes de sua ocorrência.

Nessa perspectiva, políticas de desenvolvimento social voltadas para a segurança alimentar e nutricional foram enfatizadas no Brasil a partir de 2003, como fruto de compromissos firmados internacionalmente e da maior responsabilização do Estado no enfrentamento da fome e miséria 12. Assim, diante da necessidade de conhecer a magnitude do fenômeno da insegurança alimentar e contribuir na proposição de ações para garantia da segurança alimentar e nutricional, em 2004, a Escala Brasileira de Insegurança Alimentar (EBIA) passou a integrar inquéritos populacionais realizados no país, avaliando objetivamente a experiência de fome das famílias brasileiras 13 .

Desta forma, com base nos dados da Pesquisa Nacional por Amostra de Domicílios (PNAD) dos anos de 2004, 2009 e 2013, este artigo analisou a tendência temporal e os fatores associados à insegurança alimentar no Brasil, de modo a contribuir para a compreensão desse fenômeno na década que se seguiu após a inserção da segurança alimentar e nutricional como tema prioritário na agenda política do Brasil, apontando os importantes avanços ocorridos nesse período, mas também os desafios para a superação desse problema ainda presente na sociedade brasileira.

\section{Métodos}

\section{Desenho e amostra do estudo}

A PNAD é um inquérito com amostragem probabilística complexa que possui representatividade da população brasileira residente em domicílios particulares e unidades de habitação coletivas. Para o presente trabalho, utilizamos dados secundários referentes aos inquéritos de 2004, 2009 e 2013.

O processo amostral da PNAD, delineado pelo Instituto Brasileiro de Geografia e Estatística (IBGE), foi organizado em três estágios de seleção. No primeiro, os municípios foram classificados em autorrepresentativos, com probabilidade máxima de pertencer à amostra, e não autorrepresentativos, 
em que a probabilidade foi proporcional à população residente. No segundo estágio, os setores censitários foram selecionados em cada município, de acordo com o número de unidades domiciliares. No último estágio, foram selecionados os domicílios em cada setor censitário, com equiprobabilidade para investigação das características da habitação e dos moradores, por meio de entrevistas domiciliares. $\mathrm{O}$ processo de amostragem foi o mesmo para os três inquéritos analisados, garantindo a comparabilidade dos dados 14.

Para este estudo, foram selecionados os domicílios particulares permanentes cujo módulo de segurança alimentar estava completamente preenchido e havia sido respondido por morador e que apresentavam os dados da pessoa de referência do domicílio. A amostra final foi composta por 108.377 unidades domiciliares em 2004, 117.037 em 2009 e 110.655 em 2013 (Figura 1).

\section{Variável dependente}

A insegurança alimentar no domicílio foi avaliada por meio da EBIA 15, instrumento validado e adaptado para a população brasileira a partir da escala americana elaborada, em 1990, pelo United States Department of Agriculture (USDA) 16. A EBIA é composta por 14 questões - nas quais, cada resposta afirmativa corresponde a um ponto - que avaliam a percepção e a experiência com a fome dos residentes de um domicílio nos últimos três meses, classificando-os em segurança alimentar

\section{Figura 1}

Fluxograma de seleção dos domicílios elegíveis para a análise. Pesquisa Nacional por Amostra de Domicílios (PNAD), Brasil, 2004, 2009, 2013

\begin{tabular}{|c|c|c|c|c|c|c|}
\hline \multicolumn{3}{|c|}{ Domicílios com entrevistas realizadas } & & & & \\
\hline 2004 & 2009 & 2013 & & & & \\
\hline \multirow[t]{2}{*}{112.716} & 121.163 & 116.543 & \multirow{2}{*}{ Exclusão } & \multirow{2}{*}{2004} & \multirow{2}{*}{2009} & \multirow{2}{*}{2013} \\
\hline & & & & & & \\
\hline & \multirow[b]{2}{*}{$\downarrow$} & & Domicílios coletivos & 68 & 93 & 77 \\
\hline & & & $\begin{array}{l}\text { Domicílios particulares } \\
\text { improvisados }\end{array}$ & 118 & 160 & 274 \\
\hline \multicolumn{3}{|c|}{ Domicílios particulares permanentes } & & & & \\
\hline 2004 & 2009 & 2013 & & & & \\
\hline 112.530 & 120.910 & 116.192 & Exclusão & 2004 & 2009 & 2013 \\
\hline & \multirow[b]{2}{*}{$\downarrow$} & & $\begin{array}{l}\text { Informante de segurança } \\
\text { alimentar não é morador }\end{array}$ & 4.051 & 3.756 & 5.419 \\
\hline & & & \multirow[t]{2}{*}{$\begin{array}{l}\text { Informante de segurança } \\
\text { alimentar não declarado }\end{array}$} & 51 & 42 & 23 \\
\hline \multicolumn{3}{|c|}{ Informante de segurança alimentar é morador } & & & & \\
\hline 2004 & 2009 & 2013 & & & & \\
\hline 108.428 & 117.112 & 110.750 & Exclusão & 2004 & 2009 & 2013 \\
\hline & \multirow[b]{2}{*}{$\downarrow$} & & $\begin{array}{l}\text { Ausência de dados da pessoa de } \\
\text { referência na unidade domiciliar }\end{array}$ & 51 & 50 & 44 \\
\hline & & & \multirow[t]{2}{*}{$\begin{array}{l}\text { Módulo de segurança } \\
\text { alimentar incompleto }\end{array}$} & \multirow[t]{2}{*}{0} & 25 & 51 \\
\hline \multicolumn{3}{|c|}{ Domicílios analisados } & & & & \\
\hline 2004 & 2009 & 2013 & & & & \\
\hline 108.377 & 117.037 & 110.655 & & & & \\
\hline
\end{tabular}


(0 pontos), insegurança alimentar leve (1-5 pontos na presença de moradores $<18$ anos ou 1-3 pontos na ausência de moradores < 18 anos), insegurança alimentar moderada (6-9 pontos na presença de moradores $<18$ anos ou 4-5 pontos na ausência de moradores $<18$ anos) e insegurança alimentar grave (10-14 pontos na presença de moradores < 18 anos ou 6-8 pontos na ausência de moradores $<18$ anos) 2,15 .

A condição de segurança alimentar representa a ausência de restrições alimentares na família, enquanto que, na insegurança alimentar leve, há uma preocupação com a disponibilidade de alimentos, podendo haver comprometimento qualitativo. Na forma moderada, existe uma redução na ingestão alimentar entre os adultos, e, na insegurança alimentar grave, isso atinge indivíduos menores de 18 anos e pode ocorrer a experiência da fome 2 .

\section{Variáveis independentes}

As variáveis independentes foram selecionadas considerando o modelo conceitual dos determinantes da segurança alimentar e nutricional de Kepple \& Segall-Corrêa 13. Para este estudo, foram assumidos, como determinantes distais, os indicadores localização da moradia (urbana ou rural) e as macrorregiões administrativas dicotomizadas em Norte/Nordeste e Sudeste/Sul/Centro-oeste, sendo que as últimas representam regiões de maior desenvolvimento socioeconômico 17 .

O saneamento sanitário foi considerado como determinante intermediário, categorizado como adequado quando havia acesso à rede geral de abastecimento de água, rede coletora de esgoto ou fossa séptica e coleta de resíduos sólidos; em contrapartida, considerou-se inadequado na presença de uma ou mais das seguintes condições: (a) acesso à água de poço, nascente ou outra fonte; (b) resíduos sólidos queimados, enterrados, jogados em terreno baldio, logradouro, rio, lago, mar ou outro destino; (c) esgoto destinado à fossa rudimentar, vala, rio, lago, mar ou outro; (d) ausência de banheiro ou sanitário no domicílio.

Já no nível proximal dos determinantes, incluiu-se a densidade domiciliar (razão entre o total de moradores e o número de dormitórios no domicílio) dicotomizada em $\leq 2$ moradores/dormitório; o poder econômico da família avaliado por meio do total de bens de consumo duráveis disponíveis no domicílio (geladeira de qualquer tipo, fogão de duas ou mais bocas, televisão em cores, rádio, máquina de lavar, freezer, microcomputador, filtro de água, telefone fixo e telefone celular) e categorizado em $\leq 4$ e $>4$ itens, considerando-se esse ponto de corte como número mínimo de itens necessários para a realização de atividades básicas dos indivíduos, tais como armazenamento e cocção de alimentos e comunicação, semelhantemente ao adotado por Morimoto et al. 18. Com base nessa categorização, identificamos diferença estatisticamente significante entre as médias de renda per capita em todos os anos analisados (dados não apresentados), mostrando adequação quanto à condição econômica da família, por meio de uma medida de fácil interpretação e mais estável que a renda familiar. Por fim, utilizamos os seguintes dados da pessoa referência no domicílio: sexo; faixa etária ( $\geq 60$ e $<60$ anos); raça/etnia autorrelatada (branca vs. preta, parda, amarela ou indígena); anos de estudo ( $\leq 4 \mathrm{e}>4$ anos); e condição de trabalho (empregada ou não) na semana referente à realização da pesquisa.

\section{Análise estatística}

As análises foram processadas no software Stata 12.0 (StataCorp LP, College Station, Estados Unidos), considerando-se os efeitos do plano e da ponderação do delineamento amostral complexo (survey commands) 19.

Inicialmente, as prevalências dos diferentes níveis de insegurança alimentar, com seus respectivos intervalos de 95\% de confiança (IC95\%), foram estimadas para o Brasil. Em seguida, dicotomizamos as quatro situações de insegurança alimentar em segurança alimentar + insegurança alimentar leve e insegurança alimentar moderada + insegurança alimentar grave; sendo calculado, além das proporções, o número estimado de domicílios na condição de insegurança alimentar moderada e grave na população, mediante o processo de expansão para amostras complexas. A variação temporal foi 
interpretada por meio da variação relativa da prevalência de insegurança alimentar moderada e grave entre os inquéritos de 2004 e 2013:

$$
\Delta=\frac{\left(\text { Prevalência }_{2013}-\text { Prevalência }_{2004}\right)}{\text { Prevalência }_{2004}} \times 100
$$

O processo de modelagem estatística foi conduzido a partir do modelo conceitual dos determinantes associados à segurança alimentar e nutricional 13, aplicando-se abordagem hierarquizada nas análises e utilizando o método stepwise forward para a introdução das variáveis nos modelos. A fim de produzir estimativas baseadas nas razões de prevalência (RP), com seus respectivos IC95\%, as análises foram realizadas por meio de modelos lineares generalizados especificando a função de ligação $\log \mathrm{e}$ a família de distribuição binomial.

Foram consideradas elegíveis para compor os modelos múltiplos as variáveis que apresentaram valor de $\mathrm{p}<0,20$ na análise univariada. No nível distal da análise (Modelo 1), foram consideradas as variáveis macrorregiões e localização de moradia; para a composição do Modelo 2, o saneamento foi ajustado pelas variáveis do Modelo 1; por fim, para o Modelo 3, as variáveis referentes aos determinantes proximais foram introduzidas no modelo, sendo seus efeitos ajustados pelo Modelo 2. Esse procedimento foi repetido de forma independente para cada ano analisado.

A decisão pela permanência das variáveis em cada um dos modelos foi baseada na importância do resultado para a compreensão da ocorrência da insegurança alimentar e na magnitude do efeito, bem como sua variabilidade, aqui representada pelo IC95\% 20.

\section{Ética em pesquisa com seres humanos}

Este estudo foi conduzido conforme os princípios éticos da Declaração de Helsinque, sendo dispensado de aprovação pelo Comitê de Ética em Pesquisa com Seres Humanos da Universidade Federal de Alagoas, em virtude da utilização de bases de dados de domínio público.

\section{Resultados}

No Brasil, a prevalência de segurança alimentar aumentou 19\% no período analisado, passando de 64,8\% em 2004 para 77,1\% em 2013 (Figura 2). Nesse mesmo intervalo, embora a insegurança alimentar leve tenha permanecido praticamente estável (2004: 18,2\% [IC95\%: 17,4-19,0]; 2013: 15\% [IC95\%: 13,9-16,1]), observamos redução contínua e expressiva da prevalência de insegurança alimentar moderada (2004: 10\% [IC95\%: 9,2-10,8]; 2013: 4,6\% [IC95\%: 4,2-5,1]) e de insegurança alimentar grave (2004: 7\% [IC95\%: 6,4-7,7]; 2013: 3,3\% [IC95\%: 3,0-3,6]). Contudo, as variações relativas nesses períodos não foram completamente homogêneas dentre os níveis de segurança alimentar e nutricional.

Ao compararmos os dois intervalos analisados (2004-2009 e 2009-2013), nota-se que foi no segundo período que ocorreram as maiores reduções relativas nas prevalências de insegurança alimentar leve (-20,6\%) e insegurança alimentar grave (-34\%), assim como aumento mais expressivo na segurança alimentar (10,9\%) em relação ao intervalo de 2004-2009, período que apresentou maior declínio da insegurança alimentar moderada (-34\%) (Figura 2). Especificamente quanto à insegurança alimentar moderada e grave (principal desfecho analisado), embora tenha sido possível verificar que a principal redução absoluta ocorreu no primeiro período (-5,4 vs. $-3,7$ pontos percentuais [p.p.]), do ponto de vista da variação relativa, a redução se manteve constante nos intervalos cobertos pelos três inquéritos ( 30\%) (Tabela 1).

Adicionalmente, identificamos redução sistemática na prevalência de insegurança alimentar moderada e grave em todas as estratificações de análise, todavia, não de forma homogênea (Tabela 1). Quanto às macrorregiões, houve redução na magnitude da diferença nas prevalências de insegurança alimentar moderada e grave entre as regiões Sudeste, Sul e Centro-oeste e Norte e Nordeste, passando de 19 para 10,2p.p., entre 2004-2013, contudo, nota-se, pela variação relativa, que o declínio foi maior nas regiões Sudeste, Sul e Centro-oeste $(-58,3 \%)$ quando comparadas às regiões Norte e Nordeste $(-50,7 \%)$ (Tabela 1). Como consequência desse padrão de redução relativa, identificamos, na análise 
Figura 2

Prevalência de segurança e insegurança alimentar em domicílios brasileiros, segundo ano de inquérito. Pesquisa Nacional por Amostra de Domicílios (PNAD), Brasil, 2004, 2009, 2013.

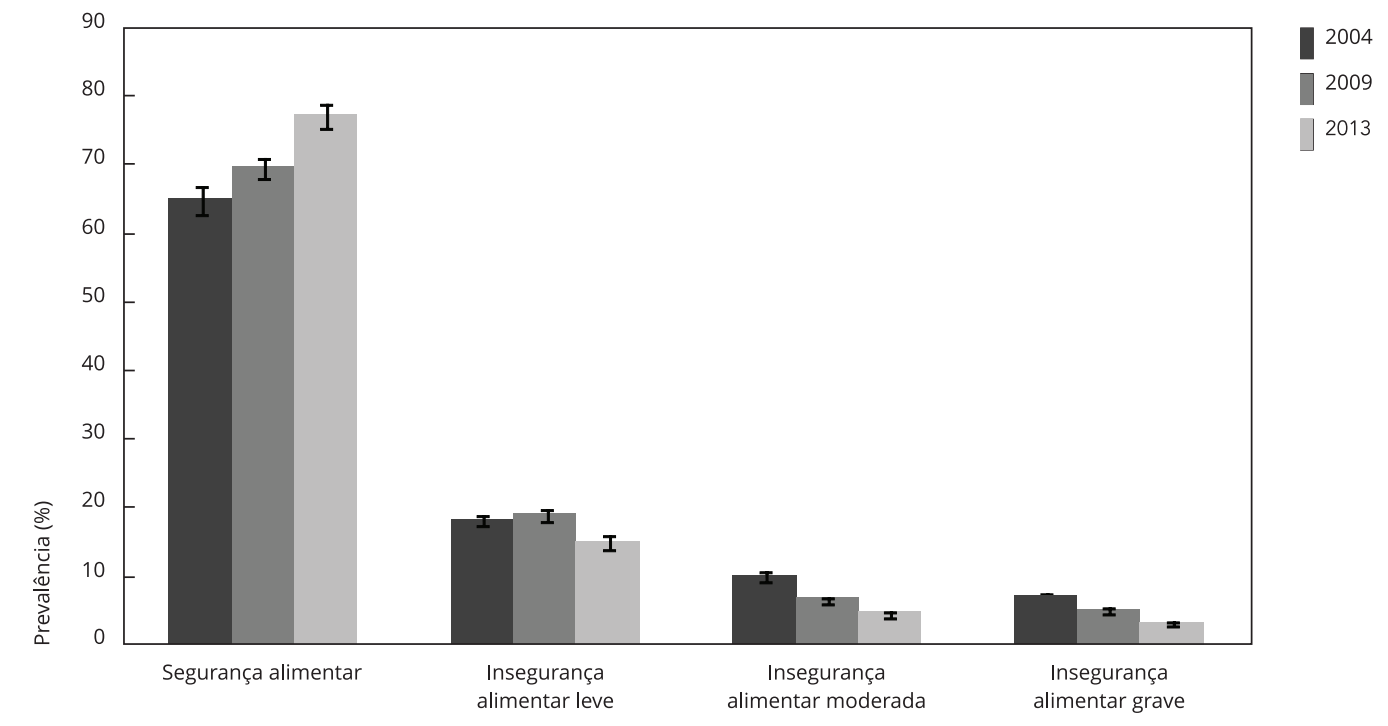

\begin{tabular}{|c|c|c|c|c|c|c|}
\hline \multirow{2}{*}{} & \multicolumn{2}{|c|}{2004} & \multicolumn{2}{c|}{2009} & \multicolumn{2}{c|}{2013} \\
\cline { 2 - 7 } & $\%$ & IC95\% & $\%$ & IC95\% & \% & IC95\% \\
\hline Segurança alimentar & 64,8 & $62,8-66,7$ & 69,5 & $68,0-71,1$ & 77,1 & $75,3-78,9$ \\
\hline Insegurança alimentar & 18,2 & $17,4-19,0$ & 18,9 & $18,0-19,7$ & 15,0 & $13,8-16,1$ \\
\hline Insegurança alimentar moderada & 10,0 & $9,2-10,8$ & 6,6 & $6,1-7,1$ & 4,6 & $4,2-5,1$ \\
\hline Insegurança alimentar grave & 7,0 & $6,3-7,7$ & 5,0 & $4,6-5,5$ & 3,3 & $2,9-3,6$ \\
\hline
\end{tabular}

IC95\%: intervalo de 95\% de confiança.

* Calculadas considerando as ponderações da amostra. As prevalências de segurança e insegurança alimentar estão apresentadas abaixo do gráfico com seus respectivos intervalos de $95 \%$ de confiança, também representados pelas barras verticais.

bruta, que as RP para o Norte e Nordeste aumentaram de 2,75 (IC95\%: 2,62-2,90) em 2004 para 3,25 (IC95\%: 3,08-3,42) em 2013 (Tabela 2).

Esse fenômeno desigual de redução das prevalências de insegurança alimentar modera e grave, impactando no aumento ou, em outros casos, manutenção das RP, repete-se em todos os níveis de determinação. Destaca-se o fato de que os estratos que representam situações de vulnerabilidade social, econômica e demográfica apresentaram, predominantemente, menor variação relativa após uma década. Por outro lado, numa perspectiva de equidade, o declínio na prevalência de insegurança alimentar moderada e grave foi homogêneo entre domicílios cujo sexo da pessoa de referência era masculino $(-55,1 \%)$ e feminino (-55\%), o que significou uma importante redução absoluta nas diferenças entre os sexos, embora os domicílios chefiados por mulheres ainda tenham apresentado maior probabilidade de insegurança alimentar moderada e grave em 2013 (1,37 [IC95\%: 1,32-1,42]) (Tabelas 1 e 2).

$\mathrm{Na}$ Tabela 3, são apresentados três modelos hierarquizados de regressão múltipla para cada inquérito. Todas as variáveis selecionadas a priori, correspondendo aos determinantes distais, intermediários e proximais apresentados na Tabela 2, foram consideradas elegíveis para a composição dos modelos. Por meio dessas análises, identificamos que os fatores associados com a insegurança alimentar moderada e grave em 2004 se mantiveram após uma década, havendo, em alguns casos, não 


\section{Tabela 1}

Prevalência e variação temporal da insegurança alimentar moderada e grave, segundo variáveis independentes. Pesquisa Nacional por Amostra de Domicilios (PNAD), Brasil, 2004, 2009, 2013.

\begin{tabular}{|c|c|c|c|c|}
\hline Variáveis & $\begin{array}{c}2004[n=108.377] \\
\% *(I C 95 \%)\end{array}$ & $\begin{array}{c}2009[n=117.037] \\
\% *(I C 95 \%)\end{array}$ & $\begin{array}{c}2013[n=110.655] \\
\% *(I C 95 \%)\end{array}$ & $\Delta \%$ ** \\
\hline Brasil & $17,0(15,7-18,4)$ & $11,6(10,7-12,6)$ & $7,9(7,2-8,7)$ & $-53,5$ \\
\hline \multicolumn{5}{|l|}{ Determinantes distais } \\
\hline \multicolumn{5}{|l|}{ Macrorregiões } \\
\hline Sudeste/Sul/Centro-oeste & $10,8(10,5-11,1)$ & $7,0(6,8-7,2)$ & $4,5(4,4-4,7)$ & $-58,3$ \\
\hline Norte/Nordeste & $29,8(28,5-31,2)$ & $20,9(20,0-21,8)$ & $14,7(14,1-15,2)$ & $-50,7$ \\
\hline \multicolumn{5}{|l|}{ Localização da moradia } \\
\hline Urbana & $15,8(15,4-16,1)$ & $10,9(10,6-11,1)$ & $6,9(6,7-7,1)$ & $-56,3$ \\
\hline Rural & $23,6(21,7-25,7)$ & $15,8(14,4-17,2)$ & $14,0(13,1-14,9)$ & $-40,7$ \\
\hline \multicolumn{5}{|l|}{ Determinante intermediário } \\
\hline \multicolumn{5}{|l|}{ Saneamento } \\
\hline Adequado & $11,6(11,3-11,9)$ & $8,4(8,1-8,7)$ & $5,3(5,2-5,5)$ & $-54,3$ \\
\hline Inadequado & $26,2(25,1-27,3)$ & $18,0(17,2-18,8)$ & $13,8(13,3-14,4)$ & $-47,3$ \\
\hline \multicolumn{5}{|l|}{ Determinantes proximais } \\
\hline \multicolumn{5}{|l|}{ Densidade domiciliar } \\
\hline$\leq 2$ pessoas/dormitório & $13,6(13,2-13,9)$ & $9,5(9,2-9,8)$ & $6,8(6,6-7,0)$ & $-50,0$ \\
\hline > 2 pessoas/dormitório & $28,7(27,8-29,7)$ & $21,5(20,8-22,3)$ & $14,5(14,0-15,1)$ & $-49,5$ \\
\hline \multicolumn{5}{|l|}{ Bens de consumo *** } \\
\hline$>4$ itens & $9,9(9,7-10,1)$ & $8,5(8,2-8,7)$ & $6,0(5,9-6,2)$ & $-39,4$ \\
\hline$\leq 4$ itens & $35,9(34,6-37,2)$ & $29,3(27,9-30,8)$ & $24,6(23,6-25,5)$ & $-31,5$ \\
\hline \multicolumn{5}{|l|}{ Sexo da pessoa de referência } \\
\hline Masculino & $15,6(15,0-16,1)$ & $10,3(9,9-10,6)$ & $7,0(6,7-7,2)$ & $-55,1$ \\
\hline Feminino & $21,1(20,6-21,6)$ & $14,4(14,0-14,7)$ & $9,5(9,2-9,8)$ & $-55,0$ \\
\hline \multicolumn{5}{|c|}{ Faixa etária da pessoa de referência (anos) } \\
\hline$\geq 60$ & $15,8(15,2-16,4)$ & $10,1(9,6-10,5)$ & $7,2(6,9-7,4)$ & $-54,4$ \\
\hline$<60$ & $17,3(16,8-17,8)$ & $12,1(11,8-12,4)$ & $8,2(7,9-8,4)$ & $-52,6$ \\
\hline \multicolumn{5}{|c|}{ Raça/Etnia da pessoa de referência } \\
\hline Branca & $10,3(10,0-10,7)$ & $6,7(6,4-6,9)$ & $4,2(4,0-4,4)$ & $-59,2$ \\
\hline Diferente de branca & $24,6(23,8-25,4)$ & $16,4(15,9-16,9)$ & $11,2(10,8-11,5)$ & $-54,5$ \\
\hline \multicolumn{5}{|c|}{ Escolaridade da pessoa de referência (anos de estudo) } \\
\hline$>4$ & $10,0(9,8-10,2)$ & $7,4(7,2-7,6)$ & $5,1(4,9-5,2)$ & $-49,0$ \\
\hline$\leq 4$ & $25,0(24,2-25,9)$ & $17,9(17,3-18,6)$ & $13,2(12,8-13,6)$ & $-47,2$ \\
\hline \multicolumn{5}{|c|}{ Condição de trabalho da pessoa de referência } \\
\hline Empregada & $15,8(15,3-16,3)$ & $10,6(10,3-11,0)$ & $7,1(6,8-7,3)$ & $-55,1$ \\
\hline Desempregada & $20,6(20,2-21,1)$ & $14,1(13,7-14,6)$ & $9,8(9,5-10,1)$ & $-52,4$ \\
\hline
\end{tabular}

IC95\%: intervalo de 95\% de confiança.

* Calculadas considerando as ponderações da amostra;

** Variação temporal relativa 2004-2013 = (((prevalência t2 - prevalência t1)/prevalência t1) X 100);

*** Diversidade de bens de consumo, avaliada conforme o número de itens distintos disponíveis no domicílio.

apenas a manutenção, mas também o aumento na força das associações, como é o caso das macrorregiões (Norte e Nordeste), saneamento (inadequado), sexo (feminino), faixa etária ( $<60$ anos), raça/ etnia (diferente de branca) e escolaridade ( $\leq 4$ anos).

Ainda na Tabela 3, destacamos a inversão da associação ocorrida em relação à localização de moradia, após a introdução da variável de saneamento (Modelo 2), que se repetiu em 2004 e 2009. Porém, no ano de 2013, a localização rural de moradia teve seu nível de associação atenuado com a introdução da variável de saneamento (1,08 [IC95\%: 1,01-1,15]), tornando-se fator de proteção para a insegurança alimentar moderada e grave (0,88 [IC95\%: 0,83-0,93]) apenas no Modelo 3, com a introdução das 
Tabela 2

Fatores associados à insegurança alimentar moderada e grave, segundo variáveis independentes (modelo linear generalizado simples). Pesquisa Nacional por Amostra de Domicílios (PNAD), Brasil, 2004, 2009, 2013

\begin{tabular}{|c|c|c|c|}
\hline Variáveis & $\begin{array}{c}2004 \text { [n = 108.377] } \\
\text { RP (IC95\%) }\end{array}$ & $\begin{array}{c}2009[n=117.037] \\
\text { RP (IC95\%) }\end{array}$ & $\begin{array}{c}2013[n=110.655] \\
\operatorname{RP}(\text { IC95\%) }\end{array}$ \\
\hline \multicolumn{4}{|l|}{ Determinantes distais } \\
\hline \multicolumn{4}{|l|}{ Macrorregiões } \\
\hline Sudeste/Sul/Centro-oeste & 1,00 & 1,00 & 1,00 \\
\hline Norte/Nordeste & $2,75(2,62-2,90)$ & $2,97(2,82-3,13)$ & $3,25(3,08-3,42)$ \\
\hline \multicolumn{4}{|l|}{ Localização da moradia } \\
\hline Urbana & 1,00 & 1,00 & 1,00 \\
\hline Rural & $1,50(1,38-1,63)$ & $1,45(1,33-1,59)$ & $2,02(1,90-2,16)$ \\
\hline \multicolumn{4}{|l|}{ Determinante intermediário } \\
\hline \multicolumn{4}{|l|}{ Saneamento } \\
\hline Adequado & 1,00 & 1,00 & 1,00 \\
\hline Inadequado & $2,25(2,15-2,36)$ & $2,14(2,02-2,27)$ & $2,59(2,47-2,71)$ \\
\hline \multicolumn{4}{|l|}{ Determinantes proximais } \\
\hline \multicolumn{4}{|l|}{ Densidade domiciliar } \\
\hline$\leq 2$ pessoas/dormitório & 1,00 & 1,00 & 1,00 \\
\hline > 2 pessoas/dormitório & $2,12(2,06-2,18)$ & $2,27(2,20-2,34)$ & $2,14(2,06-2,22)$ \\
\hline \multicolumn{4}{|l|}{ Bens de consumo * } \\
\hline$>4$ itens & 1,00 & 1,00 & 1,00 \\
\hline$\leq 4$ itens & $3,64(3,52-3,76)$ & $3,46(3,31-3,63)$ & $4,08(3,92-4,24)$ \\
\hline \multicolumn{4}{|l|}{ Sexo da pessoa de referência } \\
\hline Masculino & 1,00 & 1,00 & 1,00 \\
\hline Feminino & $1,36(1,32-1,40)$ & $1,40(1,35-1,45)$ & $1,37(1,32-1,42)$ \\
\hline \multicolumn{4}{|c|}{ Faixa etária da pessoa de referência (anos) } \\
\hline$\geq 60$ & 1,00 & 1,00 & 1,00 \\
\hline$<60$ & $1,09(1,06-1,13)$ & $1,20(1,16-1,25)$ & $1,14(1,10-1,19)$ \\
\hline \multicolumn{4}{|c|}{ Raça/Etnia da pessoa de referência } \\
\hline Branca & 1,00 & 1,00 & 1,00 \\
\hline Diferente de branca & $2,38(2,31-2,46)$ & $2,45(2,37-2,54)$ & $2,66(2,54-2,77)$ \\
\hline \multicolumn{4}{|c|}{ Escolaridade da pessoa de referência (anos de estudo) } \\
\hline$>4$ & 1,00 & 1,00 & 1,00 \\
\hline$\leq 4$ & $2,51(2,43-2,59)$ & $2,42(2,32-2,52)$ & $2,60(2,52-2,70)$ \\
\hline \multicolumn{4}{|c|}{ Condição de trabalho da pessoa de referência } \\
\hline Empregada & 1,00 & 1,00 & 1,00 \\
\hline Desempregada & $1,31(1,27-1,35)$ & $1,33(1,28-1,38)$ & $1,38(1,33-1,44)$ \\
\hline
\end{tabular}

IC95\%: intervalo de 95\% de confiança; RP: razão de prevalência.

* Diversidade de bens de consumo, avaliada conforme o número de itens distintos disponíveis no domicílio.

variáveis proximais; especificamente, após testarmos todas as variáveis desse nível, identificamos que o número de bens de consumo no domicílio e a escolaridade da pessoa de referência exerceram esse efeito sobre a localização de moradia.

\section{Discussão}

No presente artigo, analisamos a tendência da insegurança alimentar no Brasil entre o período de 2004 e 2013, o qual marca a década de início da agenda prioritária de enfrentamento da situação de fome e pobreza pelo Estado por meio de ações interministeriais. 


\section{Tabela 3}

Análise hierarquizada dos fatores associados à insegurança alimentar moderada e grave, segundo variáveis independentes (modelos lineares generalizados múltiplos). Pesquisa Nacional por Amostra de Domicílios (PNAD), Brasil, 2004, 2009, 2013.

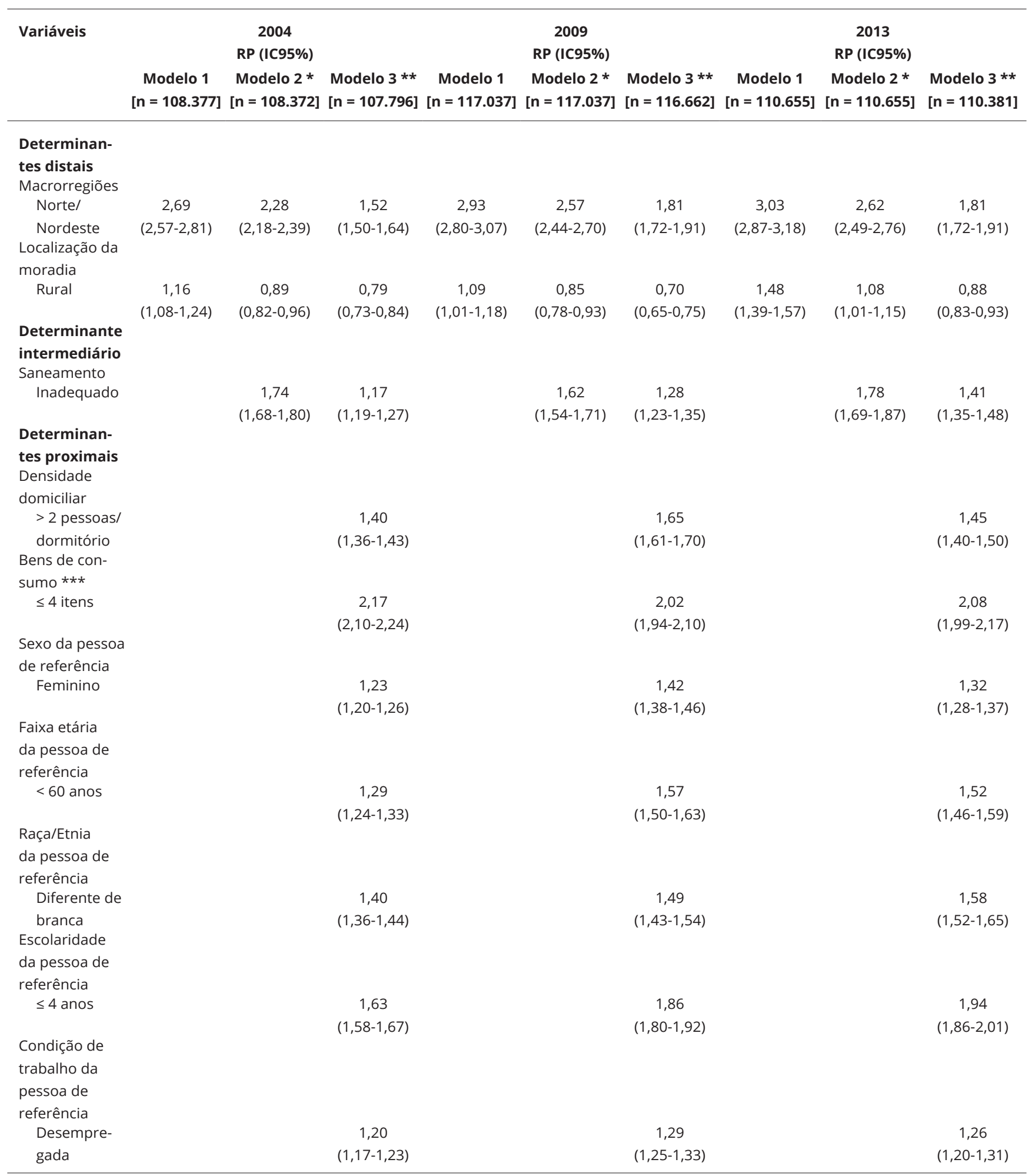

IC95\%: intervalo de 95\% de confiança; RP: razão de prevalência.

* Ajustado pelos determinantes distais (Modelo 1);

** Ajustado pelos determinantes distais (Modelo 1) e determinante intermediário (Modelo 2);

*** Diversidade de bens de consumo, avaliada conforme o número de itens distintos disponíveis no domicílio. 
A partir de nossas análises, foi possível observar redução absoluta de 9,1p.p. e redução relativa de 53,5\% na prevalência de insegurança alimentar moderada e grave no Brasil no intervalo de 2004-2013, com variação mínima de 31,5\%, nos domicílios onde os moradores possuíam $\leq 4$ bens de consumo, e máxima de 59,2\%, nos domicílios cuja pessoa de referência era branca. Além disso, identificamos que as famílias em situação de maior vulnerabilidade alimentar e nutricional foram as primeiras a se beneficiarem das políticas de promoção da segurança alimentar e nutricional, dado que a redução absoluta da insegurança alimentar moderada e grave foi mais intensa no primeiro intervalo (2004-2009).

A implantação da Estratégia Fome Zero representa o marco de referência desse processo de redução da insegurança alimentar moderada e grave observada no Brasil, a qual foi impulsionada, principalmente, pela valorização real do salário mínimo, redução do desemprego e efetivação do Programa Bolsa Família (PBF), que contribuíram para o aumento da renda familiar dos mais pobres, reduzindo os níveis de pobreza e pobreza extrema no país e, consequentemente, viabilizando maior acesso aos alimentos 21. Além disso, o investimento na agricultura familiar promoveu benefícios financeiros e técnicos aos pequenos produtores de alimentos, principais responsáveis pelo abastecimento alimentar interno no Brasil. Transversalmente a esse processo, a mobilização da sociedade civil cooperou para a institucionalização das políticas de segurança alimentar e nutricional, especialmente, a partir do restabelecimento do Conselho Nacional de Segurança Alimentar e Nutricional (CONSEA) em 200321.

Outro aspecto fundamental para o sucesso na redução da insegurança alimentar no Brasil na década analisada foi o fortalecimento progressivo das políticas de segurança alimentar e nutricional, que tiveram seu orçamento ampliado em, aproximadamente, duas vezes entre 2004-2009, passando a três vezes entre 2009-2013 22. Tais avanços também refletem o reconhecimento da alimentação como um direito constitucional e a institucionalização do Plano Nacional de Segurança Alimentar e Nutricional no ano de 2010, que passou a direcionar a implementação da política de segurança alimentar e nutricional no país 23; fatos que possivelmente estão relacionados aos maiores progressos no enfrentamento da insegurança alimentar leve e insegurança alimentar grave, acompanhados do aumento da segurança alimentar, identificados no Brasil no intervalo de 2009-2013.

Ainda assim, apesar do progresso no combate à insegurança alimentar moderada e grave, em 2013, residentes de aproximadamente 4,9 milhões de domicílios brasileiros estiveram sujeitos à condição de restrição alimentar e fome. Tal situação é inaceitável, pois se configura em violação dos direitos humanos, em que o Estado foi incapaz de garantir o acesso à alimentação adequada aos seus cidadãos, com isso, a dignidade de muitos brasileiros permanece comprometida, inviabilizando o pleno progresso social e melhores condições de vida.

Por outro lado, os resultados também apontam para uma situação complexa e desafiadora relativa à manutenção das desigualdades, uma vez que a redução da prevalência de insegurança alimentar moderada e grave foi menos intensa nos estratos populacionais com as maiores prevalências em 2004, evidenciando que as medidas adotadas para o enfrentamento da fome no Brasil não foram suficientes para promover a equidade na distribuição do fenômeno.

$\mathrm{Na}$ África do Sul, a insegurança alimentar que atingia 52,3\% da população em 1999 caiu em mais da metade (50,5\%) até 2008, assim como no Brasil, porém o declínio absoluto foi superior (26,4p.p.) 24 . Essa redução significativa reflete medidas globais de desenvolvimento socioeconômico voltadas para progressos nas condições de vida da população, tais como aumento da renda per capita mínima, benefícios de assistência social, acesso a posse de bens materiais e melhores condições de habitação 25,26,27.

Em contrapartida, observa-se, em alguns locais, o aumento da insegurança alimentar, como foi o caso de uma província canadense, onde houve aumento de $24 \%$ na probabilidade dos moradores experimentarem insegurança alimentar moderada e grave, com a prevalência passando de 7,3\% em 2005 para 8,8\% em 2012 25. Tal crescimento da insegurança alimentar moderada e grave, a partir de uma prevalência inicial de 7,3\%, remete a desafios para o enfrentamento da insegurança alimentar e da fome em situações de maior controle geral do problema e à necessidade de intervenções mais específicas dirigidas a espaços e grupos de risco. Deve-se ainda considerar o impacto de fatores como queda dos salários, desemprego, inflação dos preços e sazonalidade dos alimentos e desaceleração econômica, que têm sido associados ao aumento da insegurança alimentar em diversos contextos 25,28,29.

O Brasil é marcado historicamente por importantes desigualdades regionais, onde o Sudeste, Sul e Centro-oeste representam a metade mais rica do país. Em comparação com as outras macrorregiões, 
o Norte e Nordeste possuem produto interno bruto abaixo da média nacional, maiores disparidades em relação à concentração de renda, elevado número de municípios classificados com "baixo" IDH, além das mais altas taxas de analfabetismo e desemprego do Brasil 17,30,31. Além disso, uma limitada organização social e política acerca da segurança alimentar e nutricional nos estados e municípios poderia ainda explicar não apenas a maior ocorrência da insegurança alimentar moderada e grave nas macrorregiões Norte e Nordeste, mas também o motivo pelo qual a redução foi menos expressiva do que nas regiões de maior desenvolvimento econômico 21. Essa é uma justificativa razoável, pois considerando o caráter descentralizado da Política Nacional de Segurança Alimentar e Nutricional (PNSAN), os estados e municípios assumem maiores responsabilidades para a efetivação das ações previstas na política, sendo potencializadas ou não de acordo com o nível de participação e controle social nas localidades 23 . Com base nos dados de 2013, após a expansão da amostra, percebe-se que a realidade de consumo alimentar comprometido ou fome esteve presente em cerca de três milhões de domicílios do Norte e Nordeste.

Ainda em relação às diferenças geográficas, como mostrado em nossas análises, viver em domicílios localizados em área rural representou fator de proteção para a insegurança alimentar moderada e grave no período analisado, ao considerarmos a adequação do serviço de saneamento básico, e, em 2013, adicionalmente, o número de bens de consumo no domicílio e escolaridade da pessoa de referência.

Tais achados mostram que a crescente urbanização tem colocado desafios importantes para as famílias urbanas, pois essas têm poucas opções para reduzir a dependência da renda para a garantia da alimentação. Somado a isso, a baixa oportunidade de emprego, desigualdades sociais e acesso inadequado a serviços básicos, como saneamento, saúde, educação e assistência social, poderão impactar na perpetuação de um ciclo intergeracional de poucas oportunidades, o que limitará o desenvolvimento do capital humano nesses espaços, mantendo a população impotente para superar a condição de privação do direito humano básico à alimentação saudável e adequada, podendo expor essas famílias a desvios nutricionais 32,33 .

A proteção conferida pela área rural em relação à urbana também foi observada no Paquistão, onde famílias vivendo em área rural apresentaram menor probabilidade de insegurança alimentar, sendo esse resultado justificado pelo envolvimento com a produção alimentar para autoconsumo, reduzindo a necessidade de as famílias comprarem alimentos 34 . Outra questão que pode ter favorecido a melhor situação dos domicílios rurais brasileiros foi o maior investimento em políticas públicas de incentivo à agricultura familiar, como o Programa Nacional de Fortalecimento da Agricultura Familiar (PRONAF) e o Programa de Aquisição de Alimentos (PAA), que têm, respectivamente, financiado e contribuído para o aumento dos rendimentos dos pequenos agricultores familiares 21 .

Os resultados mostraram que menor posse de bens de consumo, desemprego e baixa escolaridade da pessoa de referência no domicílio ainda são fatores associados à insegurança alimentar moderada e grave. Em pesquisa conduzida na França, a menor posse de bens de consumo foi encontrada entre famílias vivendo em insegurança alimentar, corroborando os nossos achados 26 . A razão mais provável para essa relação se refere ao fato de que o número limitado de bens de consumo representa condição de menor nível socioeconômico ${ }^{33}$, o que comprometeria o poder de compra global da família, impactando, consequentemente, na aquisição de alimentos.

O desemprego representa uma situação adversa para o acesso aos alimentos, ao considerar que o trabalho constitui a principal fonte de renda para o sustento das famílias. Neste estudo, quando comparados os domicílios em relação à situação de trabalho da pessoa de referência, observamos que, entre aqueles que estavam desempregados, a frequência de insegurança alimentar moderada e grave foi $20 \%$, $29 \%$ e $26 \%$ maior do que nos domicílios onde a pessoa de referência estava empregada nos anos de 2004, 2009 e 2013, respectivamente. Dentre o grupo de desempregados, se excluíssemos os indivíduos que, mesmo não trabalhando, recebem algum benefício social que auxilia na subsistência familiar, encontraríamos uma probabilidade ainda mais expressiva de insegurança alimentar moderar de grave.

A associação entre desemprego e insegurança alimentar também foi encontrada por outros autores 26,35. Em países europeus, cada ponto percentual de aumento do desemprego foi associado ao aumento de 0,29p.p. na insegurança alimentar (IC95\%: 0,10-0,49) 28. Portanto, estratégias que potencializam a inserção do indivíduo no mercado de trabalho, medidas de proteção social e garantia de outras necessidades essenciais são fundamentais para minimizar o impacto do desemprego sobre a 
insegurança alimentar, tornando os recursos mais estáveis para a família 28 . A relevância da estabilidade financeira reside, entre outros aspectos, na viabilização de um acesso regular e permanente aos alimentos por todos os moradores 5 . Nosso resultado parece confirmar a importância de uma fonte de renda estável, ao menos pela pessoa de referência do domicílio, uma vez que, além da questão do desemprego, identificamos maior propensão à insegurança alimentar moderada e grave entre os domicílios com pessoa de referência mais jovem em comparação àqueles com pessoa de referência de idade $\geq 60$ anos, justificada talvez pela maior estabilidade econômica em virtude dos repasses de aposentadorias e pensões.

A associação da baixa escolaridade com a insegurança alimentar moderada e grave já era esperada, uma vez que melhores níveis de educação podem determinar melhores oportunidades de inserção no mercado de trabalho, promovendo, assim, aumento da renda familiar $5 \mathrm{e}$, com isso, o acesso à alimentação. Quando a pessoa de referência no domicílio é analfabeta ou possui baixa escolaridade, sua descendência tenderá a percorrer o mesmo caminho de limitações educacionais, podendo se tornar um adulto menos produtivo, devido à falta de oportunidades para potencializar seu desenvolvimento, ficando assim sujeito a maiores dificuldades para acessar melhores empregos e salários, o que contribui para a persistência da pobreza entre as gerações 36. Além disso, a pobreza tanto gera quanto é consequência de um estado de saúde e nutrição comprometidos; assim, o enfrentamento do ciclo da pobreza também requer investimentos na alimentação e nutrição do grupo materno-infantil a fim de favorecer habilidades cognitivas e melhores níveis futuros de escolaridade e renda entre as gerações $37,38,39$.

Neste estudo, observamos que o declínio na prevalência de insegurança alimentar moderada e grave foi expressivo nos domicílios chefiados por mulheres (55\%), equiparando-se à retração observada naqueles chefiados por homens $(55,1 \%)$ e superando a tendência de decréscimo nacional $(53,5 \%)$. Esses achados podem refletir o aumento da participação da mulher no mercado de trabalho, redução do desemprego e valorização profissional, favorecendo suas condições de suprir a alimentação para os membros da família 40 . Todavia, domićlios com pessoa de referência do sexo feminino apresentaram $23 \%, 42 \%$ e $32 \%$ mais insegurança alimentar moderada e grave do que aqueles em que o homem era a pessoa de referência em 2004, 2009 e 2013, respectivamente. A maior probabilidade de insegurança alimentar moderada e grave nos domicílios chefiados por mulheres também foi encontrada por outros autores $5,27,41$.

Por outro lado, um estudo conduzido em Bangladesh não identificou associação entre o sexo da pessoa de referência no domicílio e insegurança alimentar 42 . Atribui-se a isso o fato de se tratar de um contexto marcado pela expressiva participação feminina em atividades geradoras de renda e, além disso, descreve uma realidade sociocultural que favorece a inserção da mulher no mercado de trabalho, aumentando sua capacidade de prover a alimentação da família. Desse modo, tais comparações indicam que sociedades conservadoras apresentam restrições que limitam a emancipação feminina, apontando a necessidade de ruptura desse paradigma a fim de melhorar o acesso à alimentação saudável e adequada em residências chefiadas por mulheres.

Nas regiões Nordeste e Sul do Brasil, outro estudo identificou que a renda média per capita foi aproximadamente $30 \%$ inferior quando a mulher, em comparação ao homem, era a pessoa de referência no domicílio 27 . Ainda em uma comparação entre os sexos, as mulheres permanecem como maioria no mercado informal, recebem os piores salários e costumam não ter uma complementação da renda por cônjuge quando assumem a responsabilidade da família 5,40. Os prejuízos femininos são acentuados diante do estigma social de que a mulher deve ser a responsável pelos cuidados domésticos e com os filhos, além de trabalhar 27,40,42. Tais circunstâncias repercutem em maiores dificuldades financeiras e, consequentemente, limitam a aquisição de alimentos nos domicílios chefiados por mulheres, justificando a manutenção da desigualdade de gênero quanto à insegurança alimentar moderada e grave no Brasil.

Os domicílios com maior densidade domiciliar e pessoa de referência de raça/etnia diferente de branca também foram mais propensos à insegurança alimentar moderada e grave do que outros domicílios de referência. Isso é esperado, visto que a aglomeração intradomiciliar implica em maior número de moradores para serem alimentados, independente da renda 5; e, no caso de pessoas de raça/etnia diferente de branca, apresentam maior vulnerabilidade às condições socioeconômicas inadequadas 41 . 
Os resultados evidenciam o declínio da insegurança alimentar moderada e grave no Brasil em mais de 50\%, todavia, permanece a necessidade desafiadora de redução das desigualdades sociais. As políticas e estratégias precisam ser formuladas e executadas conforme o princípio da justiça social, considerando a complexidade e especificidade dos distintos grupos e cenários brasileiros, e priorizando os mais vulneráveis, a fim de conduzir um acesso equitativo à alimentação. Não obstante, ações intersetoriais específicas são necessárias nos diferentes níveis de determinação da insegurança alimentar abrangendo o atendimento universal e adequado às necessidades essenciais, como educação, saneamento e habitação, além de geração de emprego e renda, e aumento do poder aquisitivo das famílias. Por fim, destaca-se que, no cenário atual, ações descentralizadas são fundamentais para manter a redução da prevalência de insegurança alimentar moderada e grave no país, uma vez que as características e a intensidade dos fatores condicionantes podem variar em função do contexto em que a família está inserida.

Os pontos fortes deste estudo incluem sua representatividade nacional, o uso de medida consistente, validada e objetiva de insegurança alimentar, sendo analisada em diferentes pontos do tempo, permitindo o rastreamento detalhado do fenômeno na população brasileira na década que se seguiu após a inclusão da segurança alimentar e nutricional como prioridade de Estado. No entanto, como próprio de estudos transversais, apresentamos, como limitação, a impossibilidade de estabelecer relações causais entre as variáveis analisadas. Além disso, em virtude de a EBIA basear-se na percepção e nas diferentes experiências dos entrevistados, não podemos excluir a influência do viés de memória e de uma informação diferencial de insegurança alimentar. É importante ainda considerar, na interpretação dos resultados, que os dados apresentados para o Brasil podem não refletir a realidade encontrada em níveis geográficos menos abrangentes, e que a amostra analítica exclui a população sem endereço residencial, a qual apresenta elevada probabilidade de insegurança alimentar.

\section{Conclusão}

Por meio da análise dos dados de segurança alimentar da PNAD, foi possível observar que, entre 2004 e 2013, a população brasileira vivenciou importantes reduções na prevalência de insegurança alimentar, com predomínio das maiores alterações temporais relativas no intervalo de 2009-2013. Por outro lado, embora a redução pela metade, especificamente, dos valores absolutos de insegurança alimentar moderada e grave seja o aspecto central a ser observado, dentro da perspectiva da equidade, faz-se necessário destacar que os avanços ocorreram de modo desigual, uma vez que os estratos de maior vulnerabilidade social, econômica e demográfica, que apresentaram as maiores prevalências de insegurança alimentar moderada e grave em 2004, foram os que mostraram menores alterações relativas no decorrer da década analisada.

Observamos também que os fatores associados foram os mesmos ao longo do tempo, sendo representados principalmente por condições de vida desfavoráveis. Predominou tendência de manutenção ou elevação nas forças de associação entre essas condições adversas e a insegurança alimentar moderada e grave, confirmando a permanência de disparidades geográficas e socioeconômicas no Brasil. A responsabilização social e política nas diferentes esferas de governo deve conduzir à implementação de medidas intersetoriais sobre os determinantes da insegurança alimentar moderada e grave, com foco nos grupos mais vulneráveis à violação do direito à alimentação adequada. 


\section{Colaboradores}

T. G. Santos contribuiu na concepção do estudo, realizou a análise estatística, interpretação dos resultados, redigiu o manuscrito e é responsável por todos os aspectos do trabalho. J. A. C. Silveira contribuiu na concepção do estudo, realizou a análise estatística, interpretação dos resultados, revisão crítica do conteúdo intelectual, aprovação da versão final do manuscrito e é responsável por todos os aspectos do trabalho. G. Longo-Silva e R. C. E. Menezes contribuiu na concepção do estudo, interpretação dos resultados, revisão crítica do conteúdo intelectual, aprovação da versão final do manuscrito e é responsável por todos os aspectos do trabalho. E. K. N. M. Ramires contribuiu na interpretação dos resultados, revisão crítica do conteúdo intelectual e é responsável por todos os aspectos do trabalho.

\section{Referências}

1. Brasil. Lei no 11.346 , de 15 de setembro de 2006. Cria o Sistema Nacional de Segurança Alimentar e Nutricional com vistas em assegurar o direito humano à alimentação adequada e dá outras providências. Diário Oficial da União 2006; 18 set.

2. Segall-Corrêa AM, Marin-León L, MelgarQuiñonez H, Pérez-Escamilla R. Refinement of the Brazilian Household Food Insecurity Measurement Scale: Recommendation for a 14-item EBIA. Rev Nutr 2014; 27:241-51.

3. United Nations. Transforming our world: the 2030 Agenda for Sustainable Development, 2015. http://www.un.org/ga/search/view_ doc.asp?symbol=A/RES/70/1\&Lang=E (acessado em 23/Ago/2016).

4. Food and Agriculture Organization of the United Nations; International Fund for Agricultural Development; World Food Programme. The state of food insecurity in the world 2015. Meeting the 2015 international hunger targets: taking stock of uneven progress. Rome: Food and Agriculture Organization; of the United Nations; 2015.

5. Omuemu VO, Otasowie EM, Onyiriuka U. Prevalence of food insecurity in Egor local government area of Edo State, Nigeria. Ann Afr Med 2012; 11:139-45.

\section{Agradecimentos}

À Coordenação de Aperfeiçoamento de Pessoal de Nível Superior (Capes) pela concessão da bolsa de estudos.
6. Leung CW, Epel ES, Willett WC, Rimm EB, Laraia BA. Household food insecurity is positively associated with depression among lowincome supplemental nutrition assistance program participants and income-eligible nonparticipants. J Nutr 2015; 145:622-27.

7. Poblacion AP, Marín-León L, Segall-Corrêa AM, Silveira JA, Taddei JAAC. Insegurança alimentar em domicílios brasileiros com crianças menores de cinco anos. Cad Saúde Pública 2014; 30:1067-78.

8. Ferreira HS, Souza MEDCA, Moura FA, Horta BL. Prevalência e fatores associados à Insegurança Alimentar e Nutricional em famílias dos municípios do norte de Alagoas, Brasil, 2010. Ciênc Saúde Coletiva 2014; 19:1533-42.

9. Programa das Nações Unidas para o Desenvolvimento. Relatório do Desenvolvimento Humano 2015: o trabalho como motor do desenvolvimento humano. New York: Programa das Nações Unidas para o Desenvolvimento; 2015.

10. Seligman HK, Laraia BA, Kushel MB. Food insecurity is associated with chronic disease among low-income NHANES participants. J Nutr 2010; 140:304-10. 
11. Tamiru D, Argaw A, Gerbaba M, Ayana G, Nigussie A, Belachew T. Household food insecurity and its association with school absenteeism among primary school adolescents in Jimma zone, Ethiopia. BMC Public Health 2016; 16:802.

12. Silva SP. A trajetória histórica da segurança alimentar e nutricional na agenda política nacional: projetos, descontinuidades e consolidação. Rio de Janeiro: Instituto de Pesquisa Econômica Aplicada; 2014.

13. Kepple AW, Segall-Corrêa AM. Conceituando e medindo segurança alimentar e nutricional. Ciênc Saúde Coletiva 2011; 16:187-99.

14. Instituto Brasileiro de Geografia e Estatística. Pesquisa Nacional por Amostra de Domicílios: segurança alimentar 2013. Rio de Janeiro: Instituto Brasileiro de Geografia e Estatística; 2014.

15. Pérez-Escamilla R, Segall-Corrêa AM, Kurdian Maranha L, Sampaio MMF, Marín-León L, Panigassi G. An adapted version of the U.S. Department of Agriculture Food Insecurity module is a valid tool for assessing household food insecurity in Campinas, Brazil. J Nutr 2004; 134:1923-28.

16. Radimer KL, Radimer KL. Measurement of household food security in the USA and other industrialised countries. Public Health Nutr 2002; 5:859-64.

17. Programa das Nações Unidas para o Desenvolvimento; Instituto de Pesquisa Econômica Aplicada; Fundação João Pinheiro. Desenvolvimento humano nas macrorregiões brasileiras. Brasília: Programa das Nações Unidas para o Desenvolvimento; 2016.

18. Morimoto JM, Latorre MRDO, César CLG, Carandina L, Barros MBA, Goldbaum M, et al. Fatores associados à qualidade da dieta de adultos residentes na Região Metropolitana de São Paulo, Brasil, 2002. Cad Saúde Pública 2008; 24:169-78.

19. Heeringa SG, West BT, Berglund PA. Applied survey data analysis. Boca Raton: CRC Press; 2010.

20. Gardner MJ, Altman DG. Confidence intervals rather than $P$ values: estimation rather than hypothesis testing. Br Med J (Clin Res Ed) 1986; 292:746-50.

21. Rocha C. Developments in national policies for food and nutrition security in Brazil. Dev Policy Rev 2009; 27:51-66.

22. Câmara Interministerial de Segurança Alimentar e Nutricional. Balanço das ações do Plano Nacional de Segurança Alimentar e Nutricional - PLANSAN 2012-2015. Brasília: Ministério do Desenvolvimento Social e Combate à Fome; 2014.
23. Brasil. Decreto no 7.272 , de 25 de agosto de 2010. Regulamenta a Lei no 11.346 , de 15 de setembro de 2006, que cria o Sistema Nacional de Segurança Alimentar e Nutricional com vistas a assegurar o direito humano à alimentação adequada, institui a Política Nacional de Segurança Alimentar e Nutricional, estabelece os parâmetros para a elaboração do Plano Nacional de Segurança Alimentar e Nutricional, e dá outras providências. Diário Oficial da União 2010; 26 ago.

24. Labadarios D, McHiza ZJ, Steyn NP, Gericke G, Maunder EM, Davids YD, et al. Food security in South Africa: a review of national surveys. Bull World Health Organ 2011; 89:891-9.

25. Li N, Dachner N, Tarasuk V. The impact of changes in social policies on household food insecurity in British Columbia, 2005-2012. Prev Med 2016; 93:151-8.

26. Bocquier A, Vieux F, Lioret S, Dubuisson C, Caillavet F, Darmon N. Socio-economic characteristics, living conditions and diet quality are associated with food insecurity in France. Public Health Nutr 2015; 18:2952-61.

27. Facchini LA, Nunes BP, Motta JVS, Tomasi E, Silva SM, Thumé E, et al. Insegurança alimentar no Nordeste e Sul do Brasil: magnitude, fatores associados e padrões de renda per capita para redução das iniquidades. Cad Saúde Pública 2014; 30:161-74.

28. Loopstra R, Reeves A, McKee M, Stuckler D. Food insecurity and social protection in Europe: quasi-natural experiment of Europe's great recessions 2004-2012. Prev Med 2016; 89:44-50.

29. Gregory C, Coleman-Jensen A. Do high food prices increase food insecurity in the United States? Appl Econ Perspect Policy 2013; 35:679-707.

30. dos Santos GR, Pales RC, Rodrigues SG. Desigualdades regionais no Brasil - 1991-2010. InterSciencePlace - Revista Científica Internacional 2015; 1:145-73.

31. Melo MRB. Rendimentos e desigualdade de renda no Brasil no período 2004-2012: a contribuição da renda do trabalho da mulher na redução da desigualdade [Dissertação de Mestrado]. João Pessoa: Universidade Federal da Paraíba; 2014.

32. Floro MS, Bali Swain R. Food security, gender, and occupational choice among urban low-income households. World Dev 2013; 42:89-99.

33. Walsh CM, van Rooyen FC. Household food security and hunger in rural and urban communities in the Free State Province, South Africa. Ecol Food Nutr 2015; 52:118-37. 
34. Azeem MM, Mugera AW, Schilizzi S. Living on the edge: household vulnerability to foodinsecurity in the Punjab, Pakistan. Food Policy 2016; 64:1-13.

35. Carter KN, Lanumata T, Kruse K, Gorton D. What are the determinants of food insecurity in New Zealand and does this differ for males and females? Aust N Z J Public Health 2010; 34:602-8

36. Netto Jr. JLSN, Ramalho HMB, Silva EK. Transmissão intergeracional de educação e mobilidade de renda no Brasil. Economia e Desenvolvimento 2013; 12:6-34.

37. Martorell R, Zongrone A. Intergenerational influences on child growth and undernutrition. Paediatr Perinat Epidemiol 2012; 26 Suppl 1:302-14.

38. Warsito O, Khomsan A, Hernawati N, Anwar F. Relationship between nutritional status, psychosocial stimulation, and cognitive development in preschool children in Indonesia. Nutr Res Pract 2012; 6:451-57.
39. Victora CG, Adair L, Fall C, Hallal PC, Martorell R, Richter L, et al. Maternal and child undernutrition: consequences for adult health and human capital. Lancet 2008; 371:340-57.

40. Silva Filho LA, Queiroz SN, Clementino MLM. Mercado de trabalho nas regiões metropolitanas brasileiras. Mercator 2016; 15: 37-54.

41. Souza Bittencourt L, Chaves dos Santos SM, Jesus Pinto E, Aliaga MA, Cassia Ribeiro-Silva R. Factors associated with food insecurity in households of public school students of Salvador City, Bahia, Brazil. J Health Popul Nutr 2013; 31:471-9.

42. Mallick D, Rafi M. Are female-headed households more food insecure? Evidence from Bangladesh. World Dev 2010; 38:593-605. 


\section{Abstract}

The aim of this study was to analyze trends and factors associated with food insecurity in Brazil in 2004, 2009, and 2013, using microdata from the National Household Sample Survey (PNAD). Food insecurity was assessed using the Brazilian Food Insecurity Scale. Independent variables were selected from a conceptual model of determination of food insecurity, which was also used in the elaboration of multiple generalized linear models. The results show a downward trend in food insecurity prevalence from 2004 to 2013, especially for moderate and severe food insecurity, from $17 \%$ in 2004 (95\% CI: 15.7-18.4) to 7.9\% in 2013 (95\%CI: 7.28.7). Despite important decreases in the prevalence of moderate and severe food insecurity, regardless of the level of determination, the population strata with the lowest prevalence in 2004 showed the largest relative reduction. As for factors associated with moderate and severe food insecurity, they remained the same in the ten years covered by the PNAD survey, namely: the North and Northeast regions, urban areas with inadequate sanitation, household density $>2$ persons per bedroom, $\leq 4$ household durable consumer goods, and households headed by females, individuals $<60$ years, and non-whites, $\leq 4$ years of schooling, and being unemployed. From 2004 to 2013, the prevalence of Brazilian households with moderate and severe food insecurity dropped by half, but from the perspective of equity the advances occurred unequally and were lower in strata with greater social, economic, and demographic vulnerability.

Food and Nutrition Security; Hunger; Health Surveys; Time Series Studies

\section{Resumen}

El objetivo de este estudio fue analizar la tendencia $y$ factores asociados a la inseguridad alimentaria en Brasil durante los años 2004, 2009 y 2013, utilizando microdatos de la Encuesta Nacional por Muestra de Domicilios (PNAD). La inseguridad alimentaria se evaluó mediante la Escala Brasileña de Inseguridad Alimentaria. Las variables independientes se seleccionaron a partir de modelo conceptual de determinación de la inseguridad alimentaria, siendo utilizado también para la elaboración de los modelos lineales generalizados múltiples. Los resultados describen la tendencia de reducción en la prevalencia de inseguridad alimentaria entre 2004-2013, especialmente, en cuanto a la inseguridad alimentaria moderada y grave, que pasó de un 17\% (IC95\%: 15,7-18,4) en 2004, hasta un 7,9\% (IC95\%: 7,2-8,7) en 2013. Por otro lado, a pesar de las importantes reducciones en la prevalencia de inseguridad alimentaria moderada y grave, se observó que, independientemente del nivel de determinación, los estratos poblacionales con menor prevalencia en 2004 presentaron una reducción relativa de mayor magnitud. En cuanto a los factores asociados a la inseguridad alimentaria moderada y grave, permanecieron los mismos durante los diez años cubiertos por la PNAD, siendo estos: las macrorregiones Norte/Nordeste, área urbana (con presencia de saneamiento inadecuado), densidad domiciliaria $>2$ personas/dormitorio, poseer $\leq 4$ bienes de consumo y que la persona de referencia del domicilio sea del sexo femenino, tener una edad < 60 años, de raza/etnia diferente a blanca, tener escolaridad $\leq 4$ años y estar desempleada. Entre 2004-2013, la prevalencia de domicilios brasileños en situación de inseguridad alimentaria moderada y grave disminuyó a la mitad; no obstante, dentro de la perspectiva de la equidad, se destaca que los avances se produjeron de modo desigual, siendo menores en los estratos de mayor vulnerabilidad social, económica y demográfica.

Seguridad Alimentaria y Nutricional; Hambre; Encuestas Epidemiológicas; Estudios de Series Temporales
Recebido em 24/Abr/2017

Versão final reapresentada em 28/Set/2017

Aprovado em 24/Out/2017 